\title{
FRET Studies of the Interaction of Dimeric Cyanine Dyes with DNA
}

\author{
Stephan Laib ${ }^{1}$ and Stefan Seeger ${ }^{1,2}$
}

Received September 9, 2003; revised November 24, 2003; accepted November 24, 2003

\begin{abstract}
Fluorescence Resonance Energy Transfer (FRET) is a powerful tool to determine distances between chromophores bound to macromolecules, since the efficiency of the energy transfer from an initially excited donor to an acceptor strongly depends on the distance between the two dye molecules. The structure of the noncovalent complex of double-strand DNA (dsDNA) with thiazol orange dimers (TOTO) allows FRET analysis of two intercalated chromophores. By intercalation of two different TOTO dyes we observe an energy transfer from TOTO-1 as donor and TOTO-3 as acceptor. In this manner we are able to determine the mean distance between two proximate TOTO molecules bound to dsDNA. Thus the maximum number of binding positions for this type of intercalation dyes in the dsDNA can be obtained. Furthermore the dependency of the acceptor emission on the donor concentration is analysed. The emission of TOTO-3 reaches a maximum when the acceptor-to-donor ratio is $1: 10$.
\end{abstract}

KEY WORDS: TOTO-1; TOTO-3; TOTO-DNA complex; FRET.

\section{INTRODUCTION}

After the development of the prominent homodimeric thiazol orange dye TOTO in 1992 [1], many studies have been published to explain the excellent characteristic of the noncovalent binding properties of this dye to dsDNA [2-6]. Due to the high affinity constant of the TOTODNA complex [3] and the large increase in the fluorescence quantum yield of this dye upon intercalation to dsDNA it can be applied for DNA-fragment sizing on a single molecule level [7,8]. Accurate measurements of single DNA fragment lengths require precise, stoichiometric DNA staining with the intercalating dye molecules. Therefore an accurate knowledge of the intercalation process is of great importance.

In this study, we determined the distance between two intercalated TOTO molecules by Fluorescence Res-

\footnotetext{
${ }^{1}$ Institute for Physical Chemistry, University of Zurich, Winterthurerstrasse 190, 8057 Zurich, Switzerland.

${ }^{2}$ To whom correspondence should be addressed.E-mail: s.seeger@pci. unizh.ch.
}

onance Energy Transfer measurements. FRET is widely used to determine proximities, distances, orientations and dynamic properties of biomolecular structures and its theory has been described in numerous reviews [9-11]. Generally, in this photophysical process, energy is transferred non-radiative from an initially excited donor (D) to an acceptor (A) molecule by a dipole-dipole interaction. For donor-acceptor distances in the order of 10 to $100 \AA$, Förster derived an expression for the rate constant of energy transfer $k_{\mathrm{T}}$

$$
\begin{aligned}
k_{\mathrm{T}} & =\frac{1}{\tau_{\mathrm{D}}}\left(\frac{R_{0}}{r}\right)^{6}, \\
R_{0} & =\left(\frac{8.79 \cdot 10^{23} \cdot \kappa^{2} \cdot \Phi_{\mathrm{D}} \cdot J(\lambda)}{n^{4}}\right)^{\frac{1}{6}}[\AA] .
\end{aligned}
$$

The donor lifetime in the absence of an acceptor is represented by $\tau_{\mathrm{D}}, r$ is the donor-acceptor distance and $R_{0}$ is the Förster distance at its 50\% FRET-efficiency $[12,13]$. The magnitude of $R_{0}$ is related to the dipole-dipole orientation factor $\kappa^{2}$, the refractive index $\mathrm{n}$ of the medium between the chromophores, the spectral overlap integral 
$J(\lambda)$ and $\Phi_{\mathrm{D}}$, the quantum yield of the donor in the absence of an acceptor [11]. Due to the usage of TOTO-1-iodide as donor and TOTO-3-iodide as acceptor, $\Phi_{\mathrm{D}}$ is given as 0.34 and $J(\lambda)$ can be directly calculated from the spectra. The consensus refractive index of DNA interior is known as 1.75 [14], but because of solvation effects a effective refractive index of 1.5 is used for light path in FRET [15].

A Significant uncertainty in the distance obtained by the FRET technique often represents the dipole orientation factor $\kappa^{2}$ [16]. For the structure of the TOTO-DNA complex in solution, two dye molecules are located in a FRET distance and the transition dipoles are not perpendicular with respect to each other $[2,6,17]$. In accordance with literature values, we used a $\kappa^{2}=1.25$ to reflect the parallel orientation of the chromophores expected from the structure of the DNA double helix [15]. Hence, if the absorption spectrum of the acceptor overlaps the donor emission spectrum, the strong distance-dependence of the transfer efficiency $\mathrm{E}$ can be used to calculate the mean distance between two intercalated cyanine dyes:

$$
E=\frac{R_{0}^{6}}{R_{0}^{6}+r^{6}}=1-\frac{F_{\mathrm{DA}}}{F_{\mathrm{D}}} .
$$

The transfer efficiency can be determined experimentally from the donor emission in the absence $\left(F_{\mathrm{D}}\right)$ and presence of the acceptor $\left(F_{\mathrm{DA}}\right)$, normalized to the same donor concentration $[18,19]$.

\section{EXPERIMENTAL}

The bisintercalation dyes TOTO-1-iodide and TOTO-3-iodide (TOTO-1 resp. TOTO-3, Fig. 1) were purchased from Molecular Probes, Inc. (Eugene, USA). Bacteriophage $\lambda$-DNA, 48502 base pairs (bp) in length, (Roche, Switzerland) was stored at $-20^{\circ} \mathrm{C}$.

The stock DNA concentration was verified by absorption measurements at $260 \mathrm{~nm}$ with a Perkin Elmer Lambda 900 spectrophotometer. The bp-to-dye ratio cal-

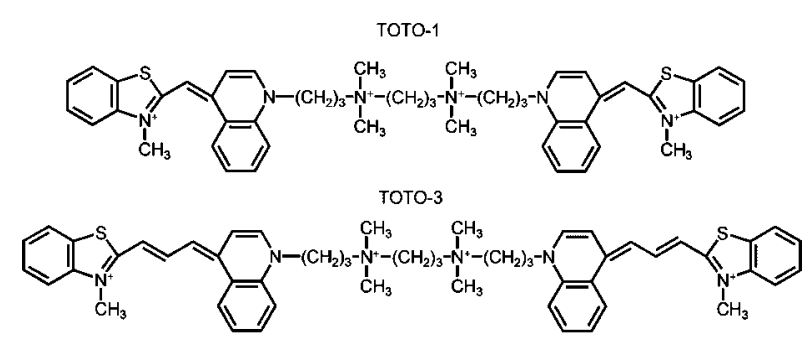

Fig. 1. Chemical structures of the dimeric thiazole orange dyes TOTO-1 and TOTO-3 used in this study. Both bisintercalators bind strongly to nucleic acids and form highly fluorescent complexes. culations were based on an average molecular mass of a deoxynucleotide base pair of $649 \mathrm{~g} / \mathrm{mol}$. DNA and dye samples were diluted in Tris/HCl buffer (10 mM Trizma Hydrochloride, $\mathrm{pH}$ 7.4, Sigma, Switzerland). To prevent the loss of TOTO-1 fluorescence by non-specific interactions between positively charged dye molecules and negatively charged phosphate backbone of DNA, $100 \mathrm{mM}$ $\mathrm{NaCl}$ was added to the solution during the distance determination measurements [20-22]. Staining solutions of DNA and TOTO-1 resp. TOTO-3 at various bp-to-dye ratios were prepared in Tris/ $\mathrm{HCl}$ buffer under reduced illumination at room temperature. The mixtures were incubated for $1 \mathrm{hr}$ prior to any measurement. All fluorescence measurements were performed on a Perkin Elmer LS 50B spectrofluorometer.

\section{RESULTS}

\section{Distance Determination Between Donor and Acceptor}

The absorption and corrected fluorescence emission spectra for TOTO- 1 and TOTO-3 intercalated to $\lambda$-DNA are shown in Fig. 2. Due to the solution structure of the TOTO-DNA complex [6] and the partial overlapping of the excitation spectrum of TOTO-3 and emission spectrum of TOTO-1, FRET can be used to determine the mean distance between two intercalated TOTO chromophores. By using the corrected emission spectrum with its area normalized to unity and $\lambda$ in centimeter units [11], a spectral overlap integral $J(\lambda)$ of $1.6 \times 10^{-16} \mathrm{~cm}^{3} \cdot \mathrm{M}^{-1}$ was calculated. Accounting for $\mathrm{n}, \kappa^{2}$ and the quantum yield of TOTO- 1 in the absence of TOTO- $3, R_{0}$ can be obtained

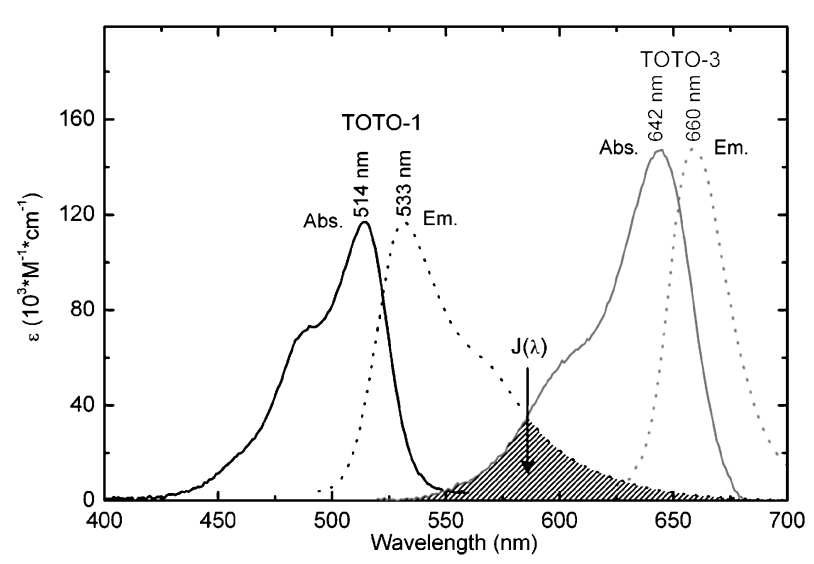

Fig. 2. Absorption (solid line) and corrected fluorescence emission spectra (dashed line) of TOTO-1 (black) and TOTO-3 (grey) in the presence of $\lambda$-DNA. The FRET spectral overlap integral $J(\lambda)$ is located between absorption spectrum of the acceptor and fluorescence emission spectrum of the donor. 


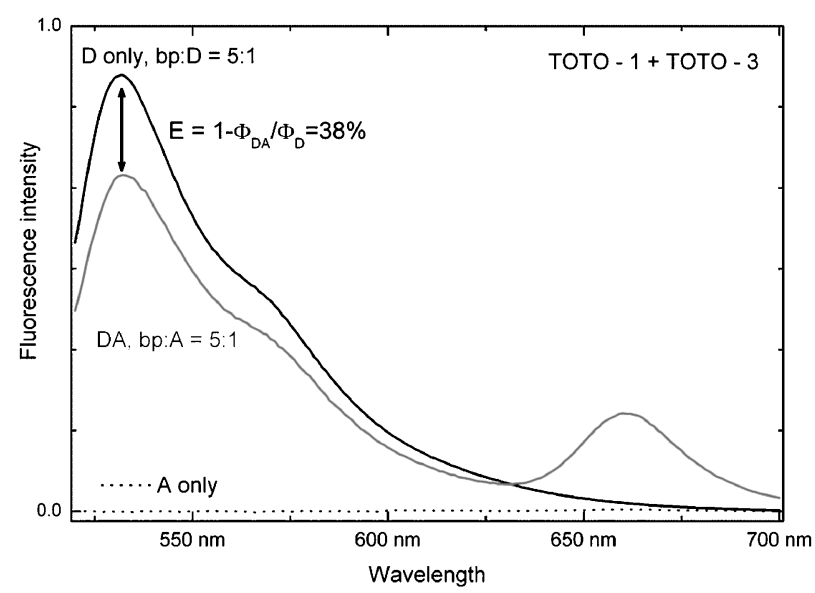

Fig. 3. Corrected fluorescence emission spectra of TOTO-1 (D, c = $0.2 \mu \mathrm{M})$ bound to dsDNA ( $\mathrm{c}=1 \mu \mathrm{Mbp})$ in the absence (black line) and presence (grey line) of TOTO-3 (A, c $=0.2 \mu \mathrm{M})$ at an excitation wavelength of $488 \mathrm{~nm}$. The dashed line shows the emission of DNA-acceptor complex without donor. The calculated energy transfer efficiency $\mathrm{E}$ is $38 \pm 2 \%$.

as $15.1 \pm 3.3 \AA$. In order to determine the transfer efficiency and the mean distance between donor and acceptor, $1 \mu \mathrm{Mbp} \lambda$-DNA solution was stained separately with $0.2 \mu \mathrm{M}$ TOTO- 1 respectively $0.2 \mu \mathrm{M}$ TOTO- 3 and with a mixture of both dyes, each with a concentration of $0.2 \mu \mathrm{M}$. Figure 3 shows the fluorescence emission spectra at an excitation wavelength of $488 \mathrm{~nm}$. Double intercalation with the mixed TOTO dyes results in a $38 \pm 2 \%$ decrease of the emission intensity at $533 \mathrm{~nm}$ compared to TOTO- 1 only stained DNA. At $660 \mathrm{~nm}$ an additional emission peak of TOTO-3 appears (cp. Fig. 2). As the reference DNA solution stained only with $0.2 \mu \mathrm{M}$ acceptor showed no fluorescence emission at $488 \mathrm{~nm}$ excitation it is evident that an energy transfer between both intercalated cyanine dyes occurred. Varying the bp-to-dye ratios between 5:1 and 1:5 leads to the same transfer efficiency. Using $R_{0}=15.1 \pm 3.3 \AA$ and the measured transfer efficiency, a mean distance between donor and acceptor of $16.4 \pm 3.6 \AA$ resp. $4.8 \pm 0.9$ bp can be determined. This result corresponds to the neighbour exclusion principle that two binding positions adjacent to the intercalated chromophore are blocked [23,24].

\section{Dependency Between Transfer Efficiency and Donor Concentration}

In order to investigate the dependency of the energy transfer on the TOTO-1-to-TOTO-3 ratio, a fluorometric titration of acceptor intercalated dsDNA with donor was carried out. Therefore $0.4 \mu \mathrm{M}$ bp $\lambda$-DNA was

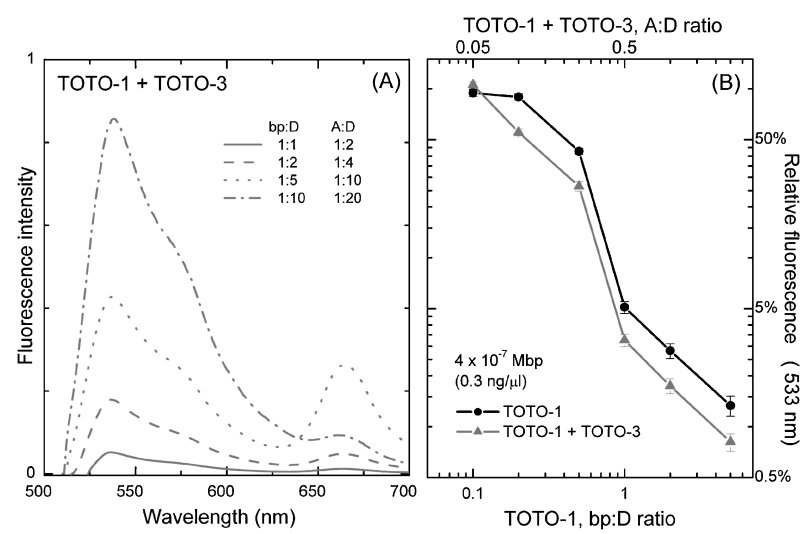

Fig. 4. Fluorometric titration of $\lambda$-DNA with TOTO- 1 (donor, D) and a constant TOTO-3 (acceptor, A) concentration. (a) Corrected fluorescence emission spectra at varied A:D ratios. The fluorescence emission of the acceptor shows a maximum when the donor concentration is 10 times higher than the acceptor concentration. (b) Fluorescence emission at $533 \mathrm{~nm}$ versus A:D ratio between 1:2.5 to 20:1. Circles represent the fluorescence emission of TOTO- 1 in the absence and triangles in the presence of TOTO-3. The efficiency of the FRET transfer is constant at $38 \pm 2 \%$ in the A:D range of $1: 2.5$ to $10: 1$.

stained with $0.2 \mu \mathrm{M}$ TOTO-3 and the donor concentration was increased step by step from 0.08 to $4 \mu \mathrm{M}$, yielding donor-to-acceptor ratios between 1:2.5 and 20:1. Simultaneously a reference solution without acceptor was titrated with the same amount of TOTO-1. FRET can be observed at all ratios and the transfer efficiency was, except for $\mathrm{A}: \mathrm{D}=1: 20$, constantly $38 \pm 2 \%$ (Fig. 4a). At high TOTO-1 concentrations the fluorescence intensity at $533 \mathrm{~nm}$ decreases, because of quenching effects and the efficiency cannot be calculated by Eq. (2) [22]. Though the amount of transferred energy is nearly constant, the fluorescence emission of the acceptor TOTO-3 at $660 \mathrm{~nm}$ increases with the amount of TOTO- 1 and reaches a maximum when the concentration of TOTO- 1 is 10 times higher than the acceptor concentration (Fig. 4b). These measurements show that it is possible to enhance the fluorescence emission of the acceptor by alteration of the amount of donor.

\section{DISCUSSION AND CONCLUSION}

\section{Distance Determination Between Donor and Acceptor}

In the well known solution structure of the complex between dsDNA and the bisintercalator TOTO the benzothiazole and quinoline rings of TOTO are both clamped by the nucleic acid base pairs and the cationic linker is placed in the minor groove. The torsional twisting around the benzothiazole/quinoline bridge is prevented in the 
complex, resulting in an increase of the fluorescence quantum yield. Here we determined the mean distance between two intercalated TOTO molecules with FRET measurements. Therefore we selected a donor acceptor pair with nearly the same solution structure: TOTO-1 and TOTO-3. Quantitative studies of the interaction of planar aromatic cations with dsDNA established two types of binding. Besides intercalation there is a weak binding mode that is characteristic of non-specific interactions of charged ligands and the phosphate groups on the DNA backbone. It is likely that externally bound dyes also perform radiationless energy transfer with the intercalated dyes. The existence of this complex depends on ionic strength and compound concentration. Consequently a formation of this non-intercalation complex is circumvented by working at salt concentrations of $0.1 \mathrm{M}$ and at compound concentrations below $100 \mu \mathrm{M}$ [21]. The resulting mean distance between two bound dyes of $4.8 \pm 0.9 \mathrm{bp}$ is consistent with the value of $4 \mathrm{bp}$ /TOTO obtained in AFM and viscometry studies [2] and corresponds to the neighbour exclusion principle [23,24].

\section{Dependency Between Transfer Efficiency and Donor Concentration}

The titration studies (Fig. 4) show that the fluorescence intensity increases with higher staining concentration of dye. According to the mass action equation, the portion of bound dye to available binding sites in DNA depends on the affinity constant and on the concentration of the educts. In respect thereof at low DNA concentrations, higher staining dye-to-bp ratios are required to obtain saturation of the DNA binding sites. In accordance with the literature [22] we reached an almost completely loaded DNA with a staining solution containing dyes and base pairs in a ratio of about 10:1.

The fluorometric titration studies (Fig. 4) proved that the fluorescence emission intensity of TOTO-3 depends on the concentration of donor, although the transfer efficiency is nearly constant within a wide range of $A: D$ ratios. If the amount of bound acceptor is less than that of donor, the TOTO-3 fluorescence emission increases. It is likely that the energy of more than one donor will be transferred to one acceptor molecule. Except at high donor concentrations the transfer efficiency can not be calculated, since the fluorescence emission of the TOTO-1 without acceptor was lost by self quenching [22].

The results of this study show that FRET measurements can be used to determine the mean distances between cyanine dyes intercalated in dsDNA. In this manner one will get worthwhile information on the binding between other intercalators, such as TO, TOTO or YOYO and DNA. Basically the maximum number of intercalated dyes per DNA fragment can be obtained. These results are useful for DNA analysis, e.g. rapid DNA-sizing methods based on the measurement of the fluorescence intensities of individual, dye intercalated DNA-fragments $[7,8]$. Furthermore the study gives an analytical approach to analyse energy migration within a DNA molecule. The results of these approaches can be helpful for studies that investigate the influences of radiation on DNA structure [25].

\section{REFERENCES}

1. H. S. Rye, S. Yue, D. E. Wemmer, M. A. Quesada, R. P. Haugland, R. A. Mathies, and A. N. Glazer (1992). Stable fluorescent complexes of double-stranded DNA with bis-intercalating asymmetric cyanine dyes-Properties and applications. Nucleic Acids Res. 20(11), 28032812.

2. J. A. Bordelon, K. J. Feierabend, S. A. Siddiqui, L. L. Wright, and J. T. Petty (2002). Viscometry and atomic force microscopy studies of the interactions of a dimeric cyanine dye with DNA. J. Phys. Chem. B 106(18), 4838-4843.

3. A. N. Glazer and H. S. Rye (1992). Stable dye-DNA intercalation complexes as reagents for high-sensitivity fluorescence detection. Nature 359(6398), 859-861.

4. N. Milanovich, M. Suh, R. Jankowiak, G. J. Small, and J. M. Hayes (1996). Binding of TO-PRO-3 and TOTO-3 to DNA: Fluorescence and hole-burning studies. J. Phys. Chem.-Us 100(21), 91819186.

5. J. P. Jacobsen, J. B. Pedersen, L. F. Hansen, and D. E. Wemmer (1995). Site-selective bis-intercalation of a homodimeric thiazole orange-dye in DNA oligonucleotides. Nucleic Acids Res. 23(5), 753760.

6. H. P. Spielmann, D. E. Wemmer, and J. P. Jacobsen (1995). Solution structure of a DNA complex with the fluorescent bis-intercalator TOTO determined by NMR-spectroscopy. Biochemistry.-Us 34(27), $8542-8553$.

7. S. Laib, M. Rankl, T. Ruckstuhl, and S. Seeger (2003). Sizing of fluorescently stained DNA-fragments by surface scanning microscopy. Nucleic Acids Res. 31(22), e138.

8. P. M. Goodwin, W. P. Ambrose, H. Cai, W. K. Grace, E. J. Larson, B. L. Marrone, J. H. Jett, J. H. Werner, and R. A. Keller (2002). in J. J. Kasianowicz, M. S. Z. Kellermayer, and D. W. Deamer (Eds.), Structure and Dynamics of Confined Polymers, Kluwer Academic, Dordecht, The Netherlands, pp. 351-370.

9. R. M. Clegg (1996). in X. F. Wang and B. Herman (Eds.), Fluorescence Imaging Spectroscopy and Microscopy, Wiley, New York, pp. 179-252.

10. L. Stryer (1978). Fluorescence energy-transfer as a spectroscopic ruler. Annu. Rev. Biochem. 47, 819-846.

11. J. R. Lakowicz (1999). Principles of Flourescence Spectroscopy, 2nd ed. Kluwer Academic/Plenum, New York.

12. T. Förster (1968). in O. Sinanoglu (Ed.), Modern Quantum Chemistry, Academic Press, New York, p. 93.

13. T. Förster (1948). Zwischenmolekulare Energiewanderung und Fluoreszenz. Ann. Phys. 2, 55-75.

14. R. E. Harrington (1970). Flow birefringence of persistence length deoxyribonucleic acid-hydrodynamic properties, optical anisotropy, and hydration shell anisotropy. J. Am. Chem. Soc. 92(23), 69576964.

15. B. P. Maliwal, J. Kusba, and J. R. Lakowicz (1995). Fluorescence energy-transfer in one-dimension-frequency-domain fluorescence study of DNA-fluorophore complexes. Biopolymers 35(2), 245255. 
16. B. W. van der Meer (2002). Kappa-squared: From nuisance to new sense. Rev. Mol. Biotechnol. 82, 181-196.

17. J. M. Schins, A. Agronskaia, B. G. de Grooth, and J. Greve (1999). Orientation of the chromophore dipoles in the TOTO-DNA system. Cytometry 37(3), 230-237.

18. J. L. Mergny, A. S. Boutorine, T. Garestier, F. Belloc, M. Rougee, N. V. Bulychev, A. A. Koshkin, J. Bourson, A. V. Lebedev, B. Valeur, N. T. Thuong, and C. Helene (1994). Fluorescence energy-transfer as a probe for nucleic-acid structures and sequences. Nucleic Acids Res. 22(6), 920-928.

19. J. R. Lakowicz, G. Piszczek, and J. S. Kang (2001). On the possibility of long-wavelength long-lifetime highquantum-yield luminophores. Anal. Biochem. 288(1), $62-75$.

20. M. T. Record, C. F. Anderson, and T. M. Lohman (1978). Thermodynamic analysis of ion effects on binding and conformational equilibria of proteins and nucleic-acids-Roles of ion association or release, screening, and ion effects on water activity. Q. Rev. Biophys. 11(2), 103-178.

21. W. D. Wilson (1999). In E. T. Kool (Ed.), DNA and Aspects of Molecular Biology, Comprehensive Natural Products Chemistry. Pergamon, Amsterdam, pp. 427-476.

22. X. M. Yan, W. K. Grace, T. M. Yoshida, R. C. Habbersett, N. Velappan, J. H. Jett, R. A. Keller, and B. L. Marrone (1999). Characteristics of different nucleic acid staining dyes for DNA fragment sizing by flow cytometry. Anal. Chem. 71(24), 54705480 .

23. D. M. Crothers (1968). Calculation of binding isotherms for heterogeneous polymers. Biopolymers 6(4), 575-580.

24. R. W. Armstrong, U. P. Strauss, and T. Kurucsev (1970). Interaction between acridine dyes and deoxyribonucleic acid. J. Am. Chem. Soc. 92(10), 3174-3180.

25. D. G. Xu and T. M. Nordlund (2000). Sequence dependence of energy transfer in DNA oligonucleotides. Biophys. J. 78(2), 1042-1058. 